\title{
Direct observation of a highly forbidden optical transition in $\mathrm{Sm}_{\mathrm{SrF}} \mathrm{Sr}_{2}$
}

\author{
Mohit Verma, Luca Talamo. ${ }^{*}$ Hiromitsu Sawaoka ${ }^{\dagger}$ and Amar C. Vutha \\ Department of Physics, University of Toronto, Canada M5S $1 A 7$
}

\begin{abstract}
The $4 f^{6}{ }^{7} F_{0} \rightarrow 4 f^{6}{ }^{5} D_{0}$ intra-configuration transition in $\mathrm{Sm}: \mathrm{SrF}_{2}$ is forbidden for $\mathrm{Sm}^{2+}$ ions in the octahedrally symmetric substitution sites in $\mathrm{SrF}_{2}$. We report the direct observation of this transition using laser-induced fluorescence at cryogenic temperatures, and measurements of the excited state lifetime and the excitation cross section. To the best of our knowledge, this optical transition has the longest lived excited state ever observed in a solid.
\end{abstract}

\section{INTRODUCTION}

Rare-earth ions (REI) doped into solid-state hosts have been studied for many decades due to their unusual optical properties [1]. More recently, REI-doped solids have been used for quantum information processing [4], and to stabilize narrow-linewidth lasers (e.g., 8 10]). REIdoped solids offer the advantage that very large numbers of atoms, typically $\gtrsim 10^{16}$ in a cm-sized crystal, can be studied without requiring laser-cooling or trapping. In addition, many applications of REI take advantage of the intra-configuration $4 f \rightarrow 4 f$ transitions in these ions, which are shielded by their closed $5 s$ and $5 p$ shells from interactions with the host lattice: this shielding leads to remarkably narrow optical spectral lines even in the solid-state [8 11]. Extremely long coherence times for electron and nuclear spins have also been demonstrated in these systems [6, 12.

The narrowest observed optical transition in a REIdoped solid thus far is the ${ }^{7} F_{0}-{ }^{5} D_{0}$ transition in $\mathrm{Eu}^{3+}$ doped into $\mathrm{Y}_{2} \mathrm{SiO}_{5}$ (YSO), with homogenous linewidth $\gamma_{h}=2 \pi \times 122 \mathrm{~Hz}$ and excited state lifetime $\tau \approx 2 \mathrm{~ms}$ at cryogenic temperatures [11, 13]. The two states involved in this clock transition have no electronic magnetic moments, and low differential sensitivity to crystal field effects. This clock transition has been applied to laser frequency stabilization using spectral hole-burning, reaching fractional frequency stabilities comparable to those achieved with high-finesse optical cavities [10, 14]. However the ${ }^{7} F_{0}-{ }^{5} D_{0}$ transition in Eu:YSO is spectrally broad, with an inhomogeneous linewidth $\Gamma_{\text {inh }} \approx 2 \pi \times 1$ $\mathrm{GHz}$ [15]. Inhomogenous broadening is typically caused by impurities and strains within the crystal that lead to ions at different locations experiencing a range of different local electromagnetic fields [10, 16, 17. Further, the nuclear magnetic dipole and electric quadrupole moments of europium open up pathways for coupling between the $\mathrm{Eu}^{3+}$ ions and their environment, including adjacent $\mathrm{Y}^{3+}$ ions in YSO 11. While such inhomogeneously broadened clock transitions are useful for laser stabilization using

\footnotetext{
* Present address: Department of Physics, University of Colorado, Boulder CO, USA.

$\dagger$ Present address: Department of Physics, Harvard University, Cambridge MA, USA.

¥ vutha@physics.utoronto.ca
}

spectral hole-burning, they cannot be used as an absolute frequency reference.

In order to explore the fundamental limits to linebroadening in such shielded REI clock transitions, we have been investigating the ${ }^{7} F_{0}-{ }^{5} D_{0}$ transition in the isoelectronic ion, $\mathrm{Sm}^{2+}$. Samarium has a number of stable isotopes, with mass numbers $A=$ $(144,147,148,149,150,152,154)$ and natural abundances of $(3,15,11.2,13.8,7.4,26.7,22.8) \%$ respectively [18. The two odd isotopes $(A=147,149)$ have nuclear spin $I=7 / 2$, while the even $A$ isotopes all have zero nuclear spin. The zero spin isotopes are particularly interesting as they could have lower inhomogeneous broadening due to the absence of nuclear moments. When doped into the $\mathrm{SrF}_{2}$ host lattice, $\mathrm{Sm}^{2+}$ (ionic radius $r_{\text {ion }}=141 \mathrm{pm} \mathrm{[19]}$ ) substitutes for the similar-sized $\mathrm{Sr}^{2+}\left(r_{\text {ion }}=140 \mathrm{pm}[20]\right)$ leading to lower doping-induced strain and potentially better isolation of the clock transition. While this system thus holds promise for realizing an optical frequency reference, the ${ }^{7} F_{0}-{ }^{5} D_{0}$ transition in $\mathrm{Sm}: \mathrm{SrF}_{2}$ had not been studied in sufficient detail prior to this investigation. The ${ }^{7} F_{0}-{ }^{5} D_{0}$ transition was only ever observed using weakly allowed decays after indirectly populating the ${ }^{5} D_{0}$ state 21, 22, and so existing estimates for its frequency were not sufficiently precise to allow continuous-wave laser excitation of this transition. The lifetime of the excited state (which sets a lower limit to $\gamma_{h}$ ) and the line strength were similarly unknown to high precision. These parameters are necessary to evaluate the feasibility of using $\mathrm{Sm}: \mathrm{SrF}_{2}$ as an optical atomic clock.

In this work, we report the first direct observation of the ${ }^{7} F_{0}-{ }^{5} D_{0}$ transition in $\mathrm{Sm}: \mathrm{SrF}_{2}$. By directly exciting the transition, we have characterized the dynamics of laser-induced fluorescence on the clock transition, and measured the excited state lifetime and the excitation cross section.

\section{APPARATUS}

The measurements were performed on a $\mathrm{Sm}: \mathrm{SrF}_{2}$ crystal (0.1\% nominal Sm concentration, no isotopic enrichment). The electronic level structure of this system is shown schematically in Fig. 1(a). The crystal was secured to the cold plate of a liquid helium cryostat using copper clamps, with indium foil used on all the metal-crystal interfaces to ensure good thermal contact. All the measurements 


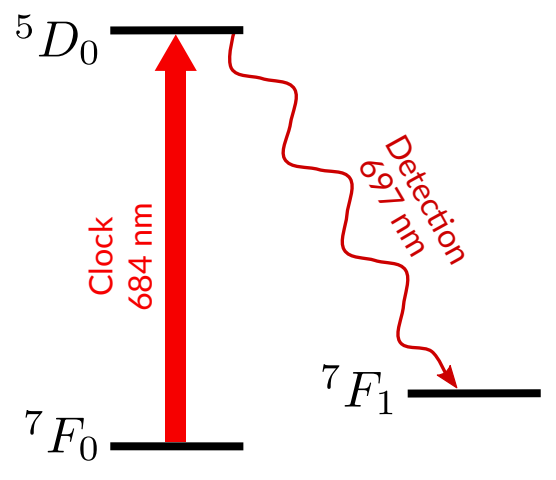

(a)

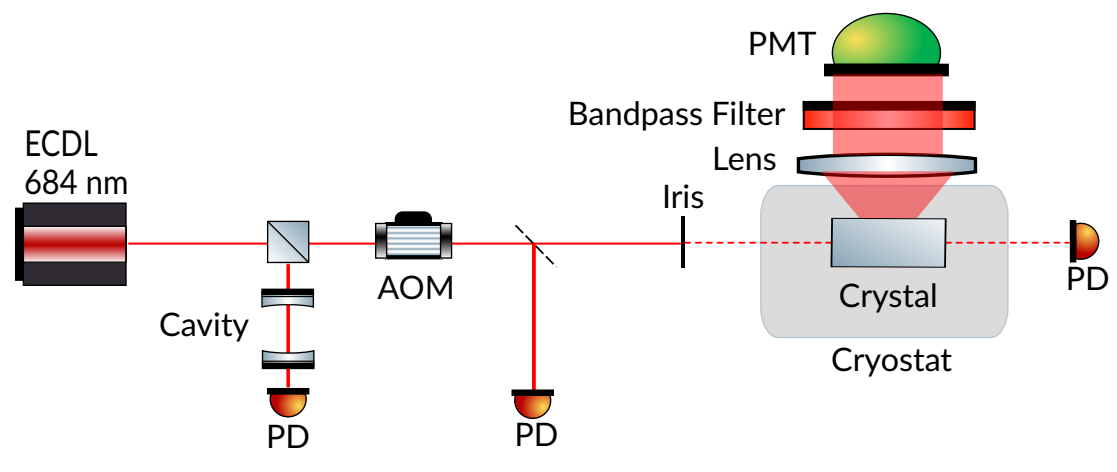

(b)

FIG. 1: (a) Schematic of the relevant energy levels of $\mathrm{Sm}^{2+}$ in $\mathrm{Sm}_{\mathrm{SrF}}$ (see Ref. 21 for a more detailed compilation). The ${ }^{7} F_{0}-{ }^{5} D_{0}$ clock transition was directly excited at $684 \mathrm{~nm}$, and detected using spontaneously emitted fluorescence from the ${ }^{5} D_{0}$ state, which primarily decays to ${ }^{7} F_{1}$ by a magnetic dipole $(M 1)$ transition at $697 \mathrm{~nm}$. All three of the energy levels shown here arise from the $4 f^{6}$ configuration. (b) Schematic of the apparatus used for the measurements reported here. ECDL is an external-cavity diode laser, AOM is an acousto-optic modulator, PMT is a photomultiplier tube, PD are photodiodes.

reported here were made at $4.2 \mathrm{~K}$, except for measurements of the temperature dependence of the excited state lifetime described below. A schematic of the apparatus is shown in Fig. 1(b).

A $684 \mathrm{~nm}$ interference-filter-stabilized external cavity diode laser (ECDL) was used to probe the clock transition. The ECDL was optimized to have a $12 \mathrm{GHz}$ scan range. The intensity of the $684 \mathrm{~nm}$ probe laser was controlled using an acousto-optic modulator (AOM), and the fluorescence signal at $697 \mathrm{~nm}$ was detected using a photomultipler tube (PMT). Light scattered into the PMT from the probe laser was suppressed using a bandpass filter centered at $697 \mathrm{~nm}$. For the cross section measurements described below, the linewidth of the probe laser was reduced to $1 \mathrm{kHz}$ by locking it to an optical cavity.

\section{MEASUREMENTS}

At the outset of this investigation, a number of conflicting values for the frequency of the ${ }^{7} F_{0}-{ }^{5} D_{0}$ transition had been reported in the literature. Wood and Kaiser 21] reported the transition wavenumber as $14616 \mathrm{~cm}^{-1}$ based on a weak emission line, whereas Gâcon et al. 23. excited ions up to the ${ }^{5} D_{0}$ state in a two-photon configuration using a pulsed laser and reported $14603 \mathrm{~cm}^{-1}$ as the transition wavenumber. Macfarlane and Meltzer [24] directly observed satellite lines at $14620 \mathrm{~cm}^{-1}$ from ions located at low-symmetry defect sites, where the transition is more strongly allowed due to admixture of energy levels by the asymmetric crystal field. After initially failing to observe the transition at $14616 \mathrm{~cm}^{-1}$ or $14603 \mathrm{~cm}^{-1}$, we were able to directly excite the transition in the neighborhood of $14612 \mathrm{~cm}^{-1}$ after a thorough search, as shown in Fig. 2, (To avoid any ambiguities between air versus vacuum wavenumbers, which are likely responsible for the

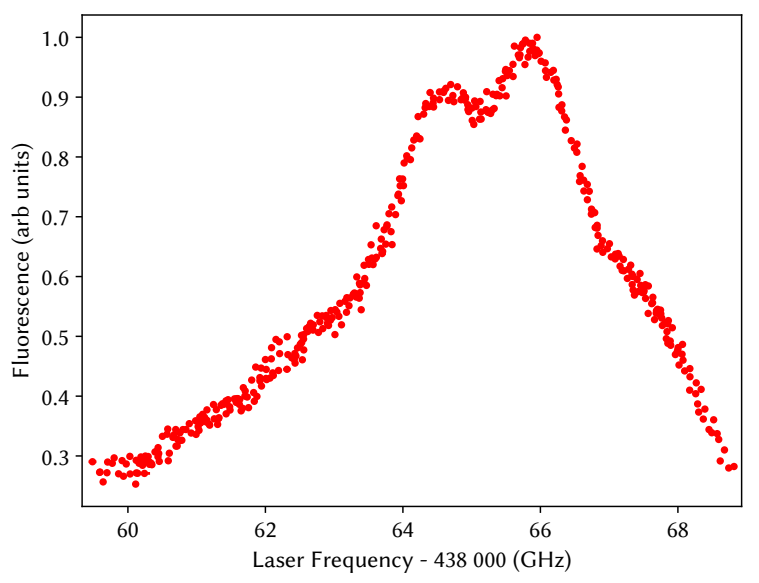

FIG. 2: The ${ }^{7} F_{0}-{ }^{5} D_{0}$ transition in $\mathrm{Sm}: \mathrm{SrF}_{2}$ observed using laser-induced fluorescence. The vertical axis shows the laser-induced fluorescence signal detected at $697 \mathrm{~nm}$, normalized to the laser power at each frequency. The

frequency axis was calibrated using a commercial wavemeter. We attribute the two peaks to inhomogeneously broadened ${ }^{7} F_{0}-{ }^{5} D_{0}$ lines from ${ }^{149} \mathrm{Sm}$

(centered at $438064.3 \mathrm{GHz}$ ) and ${ }^{147} \mathrm{Sm}$ (centered at $438065.6 \mathrm{GHz})$.

discrepancy with Ref. [21], we only use frequency units below.)

For the measurement in Fig. 2, we scanned the frequency of the $684 \mathrm{~nm}$ ECDL in $100 \mathrm{MHz}$ steps between $438056 \mathrm{GHz}$ and $438070 \mathrm{GHz}$. We used $3 \mathrm{~mW}$ of laser power and illuminated a $400 \mu \mathrm{m}$ spot ( $1 / e^{2}$ radius) along the $10 \mathrm{~mm}$ length of the crystal. The intensity of the probe laser was constant over the scan to better than 
$5 \%$, as measured using monitor photodiodes. At each frequency, 16 laser pulses (typically $50 \mathrm{~ms}$ long, repeated once every $100 \mathrm{~ms}$ ) were sent using the AOM and the 697 $\mathrm{nm}$ fluorescence decay signal was measured both during the excitation as well as in the dark. The ${ }^{5} D_{0}$ state decays almost exclusively to the ${ }^{7} F_{1}$ state, as expected for $\mathrm{Sm}^{2+}$ ions located in sites with octahedral $\left(O_{h}\right)$ symmetry [25]. A typical fluorescence time trace is shown in Fig. 3 The fluorescence signal shown in Fig. 2 was normalized to the laser power measured at each frequency using a monitor photodiode.

The fluorescence signal in Fig. 2 shows an inhomogeneously broadened zero-phonon line, which we attribute to the ${ }^{147,149} \mathrm{Sm}$ isotopes: the ratio of the heights of the two peak-like features matches the isotopic abundance ratio of ${ }^{147} \mathrm{Sm}$ and ${ }^{149} \mathrm{Sm}$ (1.09:1), and the width of these features is comparable to the inhomogeneous broadening of the ${ }^{7} F_{0}-{ }^{5} D_{0}$ transition in ${ }^{151} \mathrm{Eu}$ :YSO at similar doping concentration [13. However, the inhomogeneous profile is evidently non-gaussian, indicating that the statistical distribution of the interactions between the fluorescing ions and their local lattice environment is markedly different in $\mathrm{Sm}: \mathrm{SrF}_{2}$ compared to Eu:YSO 16. We did not detect any phonon sidebands of the transition at frequencies up to $2 \mathrm{THz}$ away from the zero-phonon line. This suggests that the ${ }^{7} F_{0}-{ }^{5} D_{0}$ transition is only weakly coupled to the $\mathrm{SrF}_{2}$ lattice.

The lifetime of the excited state was measured by fitting an exponential decay curve to the fluorescence signal measured in the dark. The decay fits very well to a single exponential, as shown in Fig. 4. The inset to Fig. 4 shows the variation of the ${ }^{5} D_{0}$ state lifetime $\tau$, as a function of the probe laser frequency. The lifetime varies by $\sim 10 \%$ over the inhomogeneously broadened profile, potentially due to variation of the matrix element for the ${ }^{5} D_{0} \rightarrow{ }^{7} F_{1}$ transition over the range of environments represented by these spectral classes. The longest lifetimes are observed near the peaks in Fig. 2. (We also measured the lifetime of the ${ }^{5} D_{0}$ state by indirectly populating it using a 410 nm laser, via the higher excited $4 f^{5} 5 d$ band, similar to the method used by Alam et al. in Ref. [22]. However, these decay curves did not fit well to single exponentials, likely due to excitation of many spectral classes from the inhomogeneous distribution in the excitation to the broad $4 f^{5} 5 d$ band - such indirect measurements of the lifetime are therefore inaccurate.) The lifetime of the ${ }^{5} D_{0}$ state measured by direct excitation of the ${ }^{7} F_{0}-{ }^{5} D_{0}$ transition (averaged across the spectral classes between 438060-438068 GHz) is $\tau=12.4(3) \mathrm{ms}$. The corresponding lower limit to the homogeneous linewidth of the transition is $\gamma_{h, \min } \approx 2 \pi \times 13 \mathrm{~Hz}$.

We also measured the lifetime of the ${ }^{5} D_{0}$ state as a function of the temperature of the crystal, as shown in Fig. 5. The lifetime fits well (albeit with a small discrepancy at low temperatures) to a simple model for a thermallyactivated decay process [22],

$$
\frac{1}{\tau}=\frac{1}{\tau_{1}}+\frac{\exp \left(-\Delta / k_{B} T\right)}{\tau_{2}},
$$

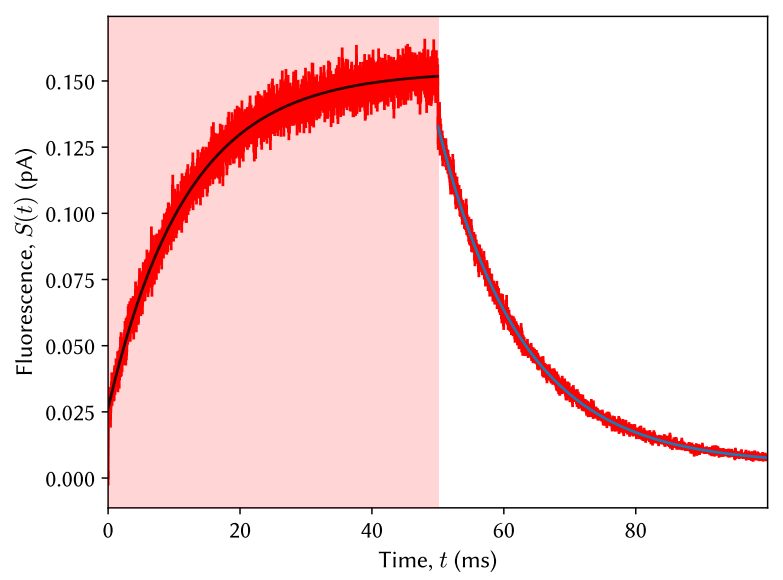

FIG. 3: Laser-induced fluorescence measured on the ${ }^{5} D_{0} \rightarrow{ }^{7} F_{1}$ line at $697 \mathrm{~nm}$. The photocathode current is plotted here as a function of time. The probe laser pulse at $684 \mathrm{~nm}$ lasts for the first $50 \mathrm{~ms}$ (shaded). The solid lines are fits to rising and falling exponentials with the same $1 / e$ lifetime $\tau$. The constant offset from residual scattered laser light is removed for clarity. The decay of the fluorescence in the dark (between 50-100 ms) from many such measurements is shown in Fig. 4.

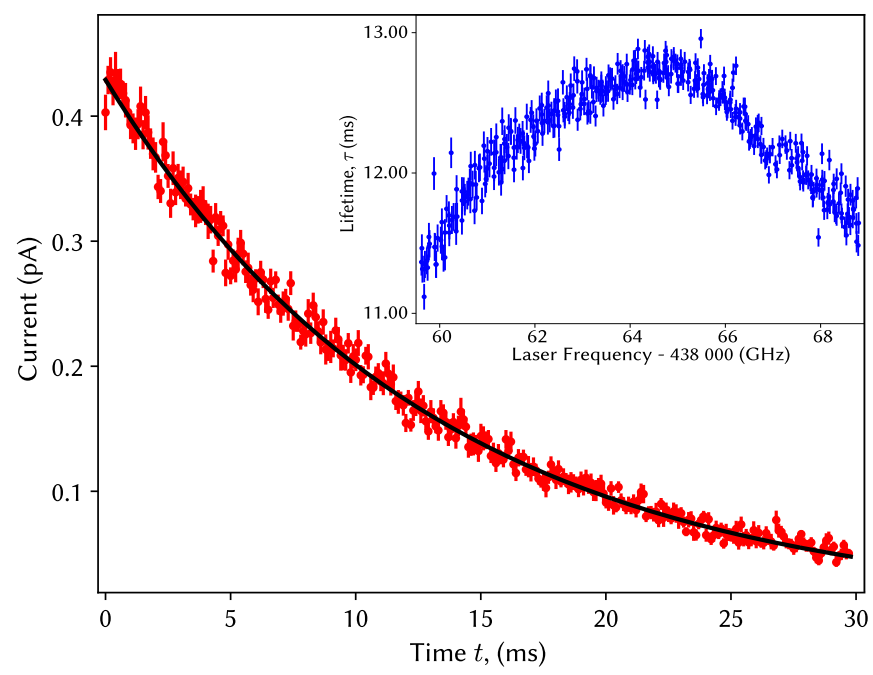

FIG. 4: The decay of fluorescence at $697 \mathrm{~nm}$ observed after pulsing the probe laser (stabilized at $438065.5 \mathrm{GHz}$ for this measurement). The solid line is a fit to a single exponential with a $1 / e$ lifetime $\tau=12.96(7) \mathrm{ms}$. The inset shows $\tau$ for different spectral classes within the inhomogeneous profile.

where $\tau_{1}$ is the lifetime of the ${ }^{5} D_{0}$ state at absolute zero, and $\tau_{2}$ is the lifetime of a shorter-lived state lying at an energy $\Delta$ above the ${ }^{5} D_{0}$ state. The fit shown in Fig. 5 yields $\tau_{2}=200 \mathrm{~ns}$ and $\Delta=h \times 14.2 \mathrm{THz}$. This energy separation $\Delta$ is consistent with an experimentally observed state that is $\sim 450 \mathrm{~cm}^{-1}$ above the ${ }^{5} D_{0}$ state 


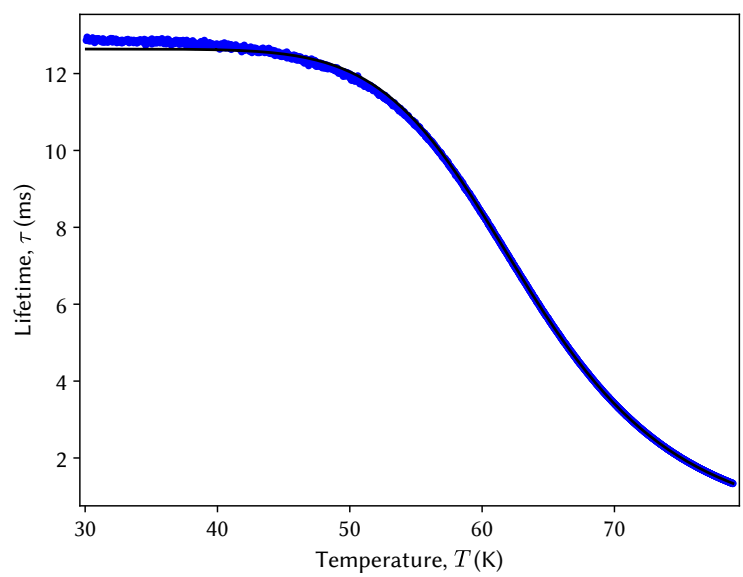

FIG. 5: The lifetime of the ${ }^{5} D_{0}$ state as a function of temperature. The solid line is a fit to the thermally-activated decay model in Eq. (1).

21].

In contrast to the ${ }^{7} F_{0}-{ }^{5} D_{0}$ transition in Eu:YSO at comparable REI densities (e.g., [26]), we did not observe any significant absorption of the probe laser through the crystal, indicating that the transition in $\mathrm{Sm}: \mathrm{SrF}_{2}$ is extremely weak. To quantify the line strength, we measured the excitation cross section for the ${ }^{7} F_{0}-{ }^{5} D_{0}$ transition as follows. Using a frequency-stabilized laser at $\nu=438066.1 \mathrm{GHz}$, we pulsed the probe laser and measured both the rise and decay of the ${ }^{5} D_{0} \rightarrow{ }^{7} F_{1}$ fluorescence as shown in Fig. 3. The exponential decay yields the lifetime $\tau$ of the ${ }^{5} D_{0}$ state as described above. The rising exponential was fit to the function $S(t)=$ $S_{0}\left(1-e^{-t / \tau}\right)+b$ to extract the steady-state fluorescence signal $S_{0}$. We then repeated these measurements at a number of values of the laser intensity $I$ (using a beam with $100 \mu \mathrm{m} 1 / \mathrm{e}^{2}$ radius), to obtain $S_{0}(I)$ as shown in Fig. 6. The nonlinearity in Fig. 6 indicates the onset of saturation on the ${ }^{7} F_{0}-{ }^{5} D_{0}$ transition.

We modeled the intensity dependence of the steadystate fluorescence signal $S_{0}(I)$ using a set of rate equations for the three energy levels shown in Fig. 11(a),

$$
\frac{d}{d t}\left(\begin{array}{c}
N_{g} \\
N_{e} \\
N_{f}
\end{array}\right)=\left(\begin{array}{c}
-\left(N_{g}-N_{e}\right) \Gamma_{\mathrm{exc}}+\gamma_{f} N_{f} \\
\left(N_{g}-N_{e}\right) \Gamma_{\mathrm{exc}}-\gamma_{e} N_{e} \\
\gamma_{e} N_{e}-\gamma_{f} N_{f}
\end{array}\right) .
$$

Here $N_{g}, N_{e}, N_{f}$ are the populations in the ${ }^{7} F_{0},{ }^{5} D_{0},{ }^{7} F_{1}$ states respectively. The excitation rate from ${ }^{7} F_{0} \rightarrow{ }^{5} D_{0}$ is $\Gamma_{\text {exc }}=\Phi \sigma$, where $\Phi=I / h \nu$ is the photon flux and $\sigma$ is the excitation cross section of interest. The decay rates for the ${ }^{5} D_{0} \rightarrow{ }^{7} F_{1}$ and ${ }^{7} F_{1} \rightarrow{ }^{7} F_{0}$ transitions are $\gamma_{e}$ and $\gamma_{f}$ respectively. $N=N_{g}+N_{e}+N_{f}$ is the total number of ions that are resonant with the laser.

The measured steady-state fluorescence $S_{0}$ is related to the ${ }^{5} D_{0}$ population at steady state, $N_{e, \text { ss }}$, via the decay rate $\gamma_{e}$, and an experimental efficiency factor $\eta_{c}$ (which

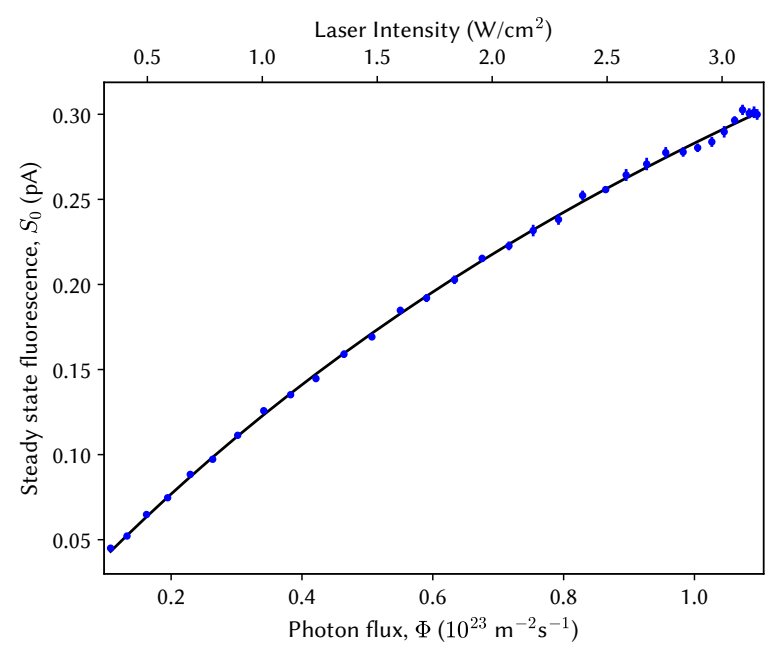

FIG. 6: The steady state fluorescence signal plotted against laser intensity. The error bars are the standard deviations of 16 repeated measurements of $S_{0}$ for each value of $I$. The solid line is a fit to Eq.(3), which yields the excitation cross section.

accounts for the solid-angle subtended by the detector, the detector's efficiency, etc.). The resulting relation between $S_{0}$ and the cross section $\sigma$ is

$$
S_{0}=\eta_{c} \gamma_{e} N_{e, \mathrm{ss}}=\eta_{c} \gamma_{e} N \frac{\Phi \sigma}{\Phi \sigma\left(2+\frac{\gamma_{e}}{\gamma_{f}}\right)+\gamma_{e}} .
$$

We fix $\gamma_{e}=1 / \tau$ to its measured value, and assume that $\gamma_{f} \gg \gamma_{e}$, since the ${ }^{7} F_{1}$ state is expected to relax quickly to the ground state due to the strong overlap between its energy above the ground state $(h \times 7.8 \mathrm{THz})$ and the phonon spectrum in $\mathrm{SrF}_{2}$ 27. This assumption is borne out by the excellent fit of Eq. (3) to the data in Fig. 6 (reduced $\chi^{2}=0.98$ ). The fit also yields a value for $\eta_{c} N$ which is consistent with an independent estimate of this quantity based on the calculated number of ions within the laser excitation volume that are resonant with the laser, manufacturer specifications for the filter transmissions and detector efficiency, and Monte Carlo modeling of the solid angle for light collection. The resulting excitation cross section is $\sigma=1.9(1) \times 10^{-18} \mathrm{~cm}^{2}$, where the uncertainty is the quadrature sum of the fit uncertainty and the estimated intensity calibration uncertainty. This small cross section confirms the highly forbidden nature of the ${ }^{7} F_{0}-{ }^{5} D_{0}$ transition in $\mathrm{Sm}: \mathrm{SrF}_{2}$.

\section{DISCUSSION AND SUMMARY}

It is interesting to ask why the transition between the ${ }^{7} F_{0}$ and ${ }^{5} D_{0}$ states is allowed at all in an octahedrally symmetric substitution site in $\mathrm{SrF}_{2}$, where it ought to be completely forbidden 25. Based on the excitation cross section measured above, we suggest that the reason 
is hyperfine-induced mixing of the ${ }^{7} F_{0}$ and ${ }^{7} F_{1}$ states. In a magnetic field $\mathcal{B}_{\mathrm{n}} \sim 100 \mathrm{G}$ due to the Sm nucleus, the ${ }^{7} F_{0}$ state is perturbed to $\left|\widetilde{{ }^{7} F_{0}}\right\rangle \approx\left|{ }^{7} F_{0}\right\rangle+\xi\left|{ }^{7} F_{1}\right\rangle$, where $\xi=\left(\mu \mathcal{B}_{\mathrm{n}} / \Delta E\right)$ with $\mu=2 \mu_{B}$ being the $M 1$ matrix element between the ${ }^{7} F_{0},{ }^{7} F_{1}$ states and $\Delta E=h \times 7.8$ $\mathrm{THz}$ their energy separation. This leads to a hyperfineinduced mixing amplitude $\xi \sim 4 \times 10^{-5}$, and an estimated homogeneous excitation cross section $\sigma_{\text {hyp }}=\xi^{2} \frac{\lambda^{2}}{2 \pi} \sim$ $10^{-18} \mathrm{~cm}^{2}$ which is comparable to the experimentally measured cross section.

The above estimate lends further credence to our suggestion that the inhomogeneously broadened lines shown in Fig. 2 arise from the ${ }^{147,149} \mathrm{Sm}$ isotopes that have nonzero nuclear magnetic moments. Despite this inhomogeneous broadening, it may be possible to use the ${ }^{147,149} \mathrm{Sm}$ lines for laser stabilization using spectral hole-burning similar to Eu:YSO, although burning persistent spectral holes in $\mathrm{Sm}: \mathrm{SrF}_{2}$ will require significantly higher optical intensities compared to Eu:YSO due to the relative weakness of the transition.

We expect that the zero spin isotopes of Sm will exhibit lower inhomogeneous broadening due to their reduced interactions with the lattice, although the clock transition in these isotopes will be extremely forbidden in the absence of hyperfine-induced mixing. The observation of the zero spin isotope lines is challenging for this reason, but is necessary in order to realize an absolute frequency reference using the $\mathrm{Sm}: \mathrm{SrF}_{2}$ system. The observation of these lines may be possible using isotopically enriched samples, or large magnetic fields to admix the ${ }^{7} F_{0},{ }^{7} F_{1}$ states. We are exploring both these approaches.

In summary, we have directly observed the highly forbidden ${ }^{7} F_{0}-{ }^{5} D_{0}$ transition in $\mathrm{Sm}: \mathrm{SrF}_{2}$. The population dynamics of the clock states under laser excitation have been understood quantitatively, allowing us to measure the lifetime of the excited state, and determine the mechanism that makes this transition slightly allowed. Our observation of this forbidden transition enables further studies of the properties of $\mathrm{Sm}: \mathrm{SrF}_{2}$, to evaluate its feasibility as a radically simple optical frequency reference.

Acknowledgments. - We acknowledge the contributions of Graham Edge, Shreyas Potnis, Wesley Cassidy and Harish Ramachandran to this experiment. We acknowledge helpful discussions with Jonathan Weinstein and Andrew Jayich. This investigation was supported by funding from NSERC, CFI, Canada Research Chairs and the Branco Weiss Fellowship. M.V., L.T., and H.S. acknowledge support from NSERC Undergraduate Student Research Awards.
[1] O. Rubio, J. Phys. Chem. Sol. 52, 101 (1991).

[2] B. G. Wybourne, J. Alloys Compd. 380, 96 (2004).

[3] R. Hull, J. Parisi, R. M. Osgood, H. Warlimont, G. Liu, and B. Jacquier, eds., Spectroscopic Properties of Rare Earths in Optical Materials, Springer Series in Materials Science, Vol. 83 (Springer-Verlag, Berlin/Heidelberg, 2005).

[4] C. Simon et al., Eur. Phys. J. D 58, 1 (2010).

[5] C. Thiel, T. Böttger, and R. Cone, J. Lumin. 131, 353 (2011).

[6] M. Zhong, M. P. Hedges, R. L. Ahlefeldt, J. G. Bartholomew, S. E. Beavan, S. M. Wittig, J. J. Longdell, and M. J. Sellars, Nature 517, 177 (2015).

[7] A. Dibos, M. Raha, C. Phenicie, and J. Thompson, Phys. Rev. Lett. 120, 243601 (2018).

[8] M. Nilsson and S. Kröll, Opt. Commun. 247, 393 (2005).

[9] M. J. Thorpe, L. Rippe, T. M. Fortier, M. S. Kirchner, and T. Rosenband, Nat. Photonics 5, 688 (2011).

[10] S. Cook, T. Rosenband, and D. R. Leibrandt, Phys. Rev. Lett. 114, 253902 (2015).

[11] R. W. Equall, Y. Sun, R. Cone, and R. Macfarlane, Phys. Rev. Lett. 72, 2179 (1994).

[12] P. Siyushev et al., Nat. Commun. 5, 3895 (2014).

[13] F. Könz, Y. Sun, C. Thiel, R. Cone, R. Equall, R. Hutcheson, and R. Macfarlane, Phys. Rev. B 68, 085109 (2003).

[14] D. R. Leibrandt, M. J. Thorpe, C.-W. Chou, T. M. Fortier, S. A. Diddams, and T. Rosenband, Phys. Rev. Lett. 111, 237402 (2013).

[15] A. Ferrier, B. Tumino, and P. Goldner, J. Lumin. 170, 406 (2016).

[16] A. Stoneham, Rev. Mod. Phys. 41, 82 (1969).
[17] R. Oswald, M. G. Hansen, E. Wiens, A. Y. Nevsky, and S. Schiller, Phys. Rev. A 98, 062516 (2018).

[18] G. Wasserburg, S. Jacobsen, D. DePaolo, M. McCulloch, and T. Wen, Geochimica et Cosmochimica Acta 45, 2311 (1981)

[19] Y. Jia, J. Solid State Chem. 95, 184 (1991).

[20] R. D. Shannon, Acta Crystallographica A 32, 751 (1976)

[21] D. Wood and W. Kaiser, Phys. Rev. 126, 2079 (1962).

[22] A. M. Alam and B. Di Bartolo, Phys. Lett. A 25, 157 (1967).

[23] J.-C. Gâcon, G. Burdick, B. Moine, and H. Bill, Phys. Rev. B 47, 11712 (1993).

[24] R. Macfarlane and R. Meltzer, Opt. Commun. 52, 320 (1985).

[25] P. A. Tanner, Chem. Soc. Rev. 42, 5090 (2013).

[26] R. Yano, M. Mitsunaga, and N. Uesugi, Opt. Lett. 16, 1884 (1991).

[27] W. Kaiser, W. Spitzer, R. Kaiser, and L. Howarth, Phys. Rev. 127, 1950 (1962). 